
du F.L.E. (18e et 19e siècles)

\title{
Lectures de filles, lectures de garçons des auteurs français de l'époque classique dans les écoles suédoises au $19^{\mathrm{e}}$ siècle
}

Elisabet Hammar

\section{OpenEdition}

Journals

Édition électronique

URL : https://journals.openedition.org/dhfles/3022

DOI : $10.4000 /$ dhfles.3022

ISSN : 2221-4038

Éditeur

Société Internationale pour l'Histoire du Français Langue Étrangère ou Seconde

Édition imprimée

Date de publication : 1 décembre 1999

Pagination : p.187-199

ISSN : 0992-7654

Référence électronique

Elisabet Hammar, «Lectures de filles, lectures de garçons des auteurs français de l'époque classique dans les écoles suédoises au 19 e siècle », Documents pour l'histoire du français langue étrangère ou seconde [En ligne], 24 | 1999, mis en ligne le 31 janvier 2015, consulté le 27 mai 2021. URL : http:// journals.openedition.org/dhfles/3022 ; DOI : https://doi.org/10.4000/dhfles.3022

Ce document a été généré automatiquement le 27 mai 2021.

(c) SIHFLES 


\title{
Lectures de filles, lectures de garçons des auteurs français de l'époque classique dans les écoles suédoises au $19^{\mathrm{e}}$ siècle
}

\author{
Elisabet Hammar
}

Disons tout de suite que si on veut étudier la présence des auteurs français de l'époque classique $^{1}$ dans l'enseignement des langues des écoles en Suède avant notre siècle, et tout particulièrement si on veut centrer cette étude sur la différence de lecture entre les garçons et les filles, on est, par la force des choses, repoussé vers la fin du $19^{e}$ siècle, la matière pour une comparaison n'étant plus à notre disposition avant cette période. Ceci nous amène vers l'époque où, du moins en Suède, le nombre d'écoles de filles est devenu assez important pour que les autorités commencent à en tenir compte et aussi vers l'époque où les écoles publiques pour garçons prennent de l'ampleur et que l'enseignement des langues vivantes y occupe une place de plus en plus importante, entraînant finalement un grand débat sur les objectifs de leurs études et les méthodes à suivre pour atteindre ces objectifs. Ce débat aboutit au mouvement de la Réforme, vers les années 1880, mouvement qui n'implique pas seulement un changement dans l'attitude à l'égard de l'équilibre, dans l'apprentissage, entre la langue parlée et la langue écrite, mais aussi dans celle à l'égard de la langue à apprendre. Les langues n'étaient plus un champ d'exercices pour former l'esprit logique de l'apprenant; les textes, au lieu de fournir matière à un rébus à déchiffrer, devaient servir à des buts pratiques: donner des exemples d'une langue naturelle de tous les jours, à réutiliser à l'occasion, ainsi que montrer, de façon concrète, comment vivent et réagissent les habitants du pays en question. En outre, la langue à apprendre n'était plus celle des époques révolues, mais la langue contemporaine. La conclusion est simple: dans ce contexte, les auteurs français de l'époque classique ne devraient pas, vers la fin du siècle dernier, être à la mode et un chercheur s'efforcerait en vain d'en découvrir les traces dans les manuels et les rapports des activités des écoles. 
2 L'édition, comme toujours dans le domaine qui nous intéresse -l'histoire de l'enseignement des langues- peut nous donner des points de départ, même si elle constitue une matière incertaine en ce qui concerne l'utilisation du contenu. Un livre peut ne pas avoir été utilisé du tout ou utilisé d'une manière que nous ne pouvons pas deviner. Il est également impossible de savoir si certains textes dans un livre de lecture ou une anthologie ont vraiment été lus, et l'importation des livres étrangers, dans ce cas en Suède, était loin d'être insignifiante au cours du $19^{\mathrm{e}}$ siècle, comme au cours des siècles précédents. Le premier phénomène peut entraîner une surévaluation de la lecture de certains auteurs, le deuxième une sous-évaluation. L'édition dans un pays donné fournit pourtant indubitablement une certaine indication sur l'orientation des études et des lectures à une époque donnée, surtout si l'on tient compte de la précarité des conclusions tirées.

3 Il me semble pratique de diviser le siècle en quatre périodes: celle du début du siècle jusqu'en 1840 peut être considérée comme la préhistoire de l'enseignement des langues institutionnalisé; la période de 1840 à 1870 comme les débuts tâtonnants d'une instruction nationale; celle de 1870 à 1890 comme l'époque parfois tapageuse des mises en cause des procédés dont on s'était servi jusque là; et la dernière décennie comme la maturation des expériences faites. On voit que même au cours du siècle dernier, l'évolution va de plus en plus vite. Cette division donne des périodes de plus en plus courtes: la première compte quarante ans, la suivante trente ans, puis vingt ans, et finalement une décennie.

4 Comment donc se situent les auteurs français de l'époque classique à l'intérieur de ces périodes, dans l'édition et dans l'enseignement effectué? Peut-on discerner une différence entre la lecture des garçons et celle des filles?

5 Le siècle commence, comme le $18^{\mathrm{e}}$ se termine, sous le signe de Télémaque de Fénelon. Je ne m'y attarderai pas, puisque cet ouvrage incontournable dans l'enseignement du français dans tous les pays a été l'objet d'une autre communication dans ce colloque, à plus forte raison quand cette autre communication traite, comme celle-ci, de la situation suédoise. La $5^{\mathrm{e}}$ édition suédoise de cet ouvrage parut en 1802 , suivie de trois autres avant $1840^{2}$, ce nombre d'éditions étant exceptionnel pour la Suède. Cette mise en vente régulière, ainsi que l'arrêt de sa publication ${ }^{3}$, coïncide presque exactement avec l'étude de ce texte dans le lycée de Stockholm4.

6 Dès que l'on quitte ce texte, il devient difficile de cerner les textes de l'époque classique, pas tant dans l'édition, que dans les études faites. Par la suite, ces textes se faufilent le plus souvent dans des livres de lecture ou des anthologies, sans toujours être «signés» d'ailleurs, et, dans les rapports sur la lecture effectuée au cours de l'année dans une école donnée, on cite le titre du volume utilisé, mais rarement les pages travaillées. Avec le peu de documentation dont je dispose, je veux pourtant essayer d'en tracer une image.

7 L'édition des ouvrages plus ou moins complets des auteurs de l'époque classique, d'anthologies et de livres de lecture au cours de la période 1800-1840 se répartit de la manière suivante:

Publication d'un ouvrage plus ou moins complet:

\begin{tabular}{|l|l||l}
\hline $\begin{array}{l}\text { Avant l'époque } \\
\text { classique }\end{array}$ & $\begin{array}{l}1 \\
\text { édition }\end{array}$ & Nepos. \\
\hline
\end{tabular}




\begin{tabular}{|l|l|l|}
\hline $\begin{array}{l}\text { L'époque } \\
\text { classique }\end{array}$ & $\begin{array}{l}5 \\
\text { éditions }\end{array}$ & Télémaque, 4 éd.; Racine, Iphigénie/Athalie, 1 éd. \\
\hline $18^{e}$ siècle & $\begin{array}{l}18 \\
\text { éditions }\end{array}$ & $\begin{array}{l}\text { Marmontel, Florian, Sterne,de Genlis, Berquin, L.E. Paban, Bouilly, } \\
\text { Leprince de Beaumont, Voltaire, Ramsay, Disbrowe. }\end{array}$ \\
\hline $19^{e}$ siècle & - & \\
\hline Total: & $\begin{array}{l}24 \\
\text { éditions }\end{array}$ & \\
\hline Anthologies et livres de lecture: \\
\hline
\end{tabular}

9 Les ouvrages qui donnent très peu de textes de l'époque classique, présentent deux fables de La Fontaine (Gedike ${ }^{8}, 6$ éd., 1802-32) et quelques Caractères de La Bruyère (Moberg $\left.{ }^{9}, 1807\right)$. Le plus grand nombre de pages tiré des auteurs classiques dans l'un des recueils qui restent est occupé par Bossuet, Les Grecs, Boileau, Tableau des troubles de Paris et trois fables de La Fontaine (Svedelius ${ }^{10}, 1817$ \& 1832). Dans l'autre, les textes en prose sont dominés par Fénelon et La Bruyère, tandis que la poésie est représentée par Boileau, La Fontaine, Racine et Molière (L.E. Paban, Beautés... 1834 \& 183711).

En ce qui concerne l'utilisation des ces différents ouvrages ou des témoignages d'une lecture des auteurs classiques dans les écoles, je ne dispose pour le moment que de ce qui reste de renseignements sur les écoles de Stockholm ${ }^{12}$. Il me semble que si on prend la peine de donner des détails sur les textes lus, il s'agit surtout des auteurs classiques, sauf dans le cas d'une école de filles. Dans le seul lycée qui existait, on travaille sans relâche Télémaque jusqu'en 1835, puis les Beautés... de Paban. Dans deux écoles privées, on étudie dans l'une (Fribyggarelyceum) Télémaque, et les livres de lecture de Moberg et de Svedelius ${ }^{13}$; dans l'autre (Hillska skolan à Barnângen) "des auteurs classiques» et l'ouvrage de Svedelius ${ }^{14}$. Dans la seule école de fdles de laquelle il reste de la documentation, l'École de Wallin, fondée en 1831, on travaille pendant ces années-ci $1 \&$ Numa Pompilius de Florian ${ }^{15}$, Voltaire, Chateaubriand, Lamartine, de Vigny, mais aussi Molière ${ }^{16}$, sans aucune indication sur les œuvres dont il est question. Il est pourtant tentant de lier le nom de ces auteurs aux titres de l'édition de la période suivante, 1840-1870, qui offre le tableau suivant:

11 Publication d'un ouvrage plus ou moins complet:

\begin{tabular}{|l|l|l|}
\hline $\begin{array}{l}\text { Avant l'époque } \\
\text { classique }\end{array}$ & - & \\
\hline $\begin{array}{l}\text { L'époque } \\
\text { classique }\end{array}$ & - & \\
\hline $18^{\mathrm{e}}$ siècle & $\begin{array}{l}3 \\
\text { éditions. }\end{array}$ & Voltaire, Histoire de Charles XII, 2 éd; Bouilly \\
\hline
\end{tabular}




\begin{tabular}{|l|l|l|}
\hline $19^{\text {e }}$ siècle & $\begin{array}{l}8 \\
\text { éditions. }\end{array}$ & $\begin{array}{l}\text { Lamartine, Voyage en Orient, 2 éd; Souvenirs... ; Christophe Colomb; } \\
\text { Dumas, Hist. de Napoléon; Chateaubriand, Atala-René; Saintine, } \\
\text { Dielitz. }\end{array}$ \\
\hline Total: & $\begin{array}{l}11 \\
\text { éditions }\end{array}$ & \\
\hline \hline & Anthologies et livres de lecture: \\
\hline & $\begin{array}{l}42 \\
\text { éditions }\end{array}$ & $\begin{array}{l}13 \text { éditions contiennent des textes de l'époque classique, dont } 9 \text { dans } \\
\text { des proportions surpassant 1/10 du volume. }\end{array}$ \\
\hline
\end{tabular}

12 On croit tout de suite voir quelques tendances. Premièrement, il semble que, avec la scolarisation grandissante, la mode soit passée de l'impression des ouvrages complets à la publication d'anthologies et de livres de lecture; deuxièmement, les textes des auteurs anciens et de l'époque classique paraissent plutôt en forme d'extraits dans ces derniers. Cette impression est, certes, partiellement trompeuse, puisque trois des neuf ouvrages présentent des pièces entières de Racine (Elfving $\mathrm{II}^{17}$, 1850: Iphigénie) et de Molière (LE Paban, Nouv.bibl. $\mathrm{III}^{18}$, 1861: L'Avare; Pauli, Fr.lasbok $\mathrm{II}^{19}$, 1867: Les Fourberies de Scapin). Quatre des autres sont en revanche comme une sorte de protestation contre la «modernisation» des textes pour les écoles, puisqu'ils donnent une très forte proportion de textes provenant de l'époque classique: Caravello, Edda française ${ }^{20}, 1846$, trois quarts des textes; Dubb, Handbok....1 $\backslash 1853$ et 1859, presque la moitié des textes; et Staaf, Urval...22, 1859, consacre le premier cours du premier tome de son grand exposé sur la littérature français ${ }^{23}$ à «L'époque depuis la naissance de la littérature classique avec Malherbe jusqu'à la mort de Louis XIV» (275 pages) dont à peine une quarantaine présentent des textes qui sont à considérer comme appartenant à l'époque antérieure à ce qu'on appelle normalement l'époque classique.

13 Il s'agit donc de trois auteurs qui ont voulu faire connaître à la jeunesse suédoise les auteurs classiques: Caravello, Dubb et Staaf. Pour la présentation, ils ont choisi trois manières différentes, et couvrent en le faisant les trois procédés traditionnels pour faire ce genre d'ouvrages, un choix de textes plus ou moins littéraires. Caravello organise donc les textes sous des titres ou thèmes, dans son cas, «Fables», «Théâtre», «Mélanges en prose» et «Mélanges en vers»; Dubb suit un ordre strictement chronologique, tandis que Staaf, tout en respectant l'ordre chronologique, divise les textes en deux parties, les textes en prose et la poésie.

14 Sous «Fables», Caravello présente un peu de Florian et beaucoup de La Fontaine; sous «Théâtre», il s'agit de fragments de pièces de Molière et de Racine, finalement d'une vingtaine de pages tirées du Fanatisme de Voltaire. Pour les «Mélanges en prose» il tire quelques pages de Bossuet et de Fénelon; le reste constitue des textes pris au $18^{\mathrm{e}}$ siècle. Les vers, par contre, sont surtout tirés du $17^{\mathrm{e}}$ siècle, de L'Art poétique de Boileau et des cantiques provenant d'Athalie et d'Esther de Racine.

Dubb dispose de ses presque 200 pages d'auteurs classiques en favorisant nettement le théâtre; 40 pages de Molière avec des extraits du Misanthrope, du Tartuffe et de L'Avare; et encore 40 pages de Racine, Athalie avec sa préface. A la troisième place vient Fénelon, presque 30 pages, dont la plus grande partie constitue des extraits de Télémaque; à la quatrième Bossuet, L'Histoire universelle et deux oraisons funèbres. La Fontaine est 
représenté par 11 fables et l'Epilogue, et La Bruyère occupe 9 pages avec ses Caractères. Les auteurs à qui on donne un plus petit nombre de pages sont Boileau, Madame de Sévigné, La Rochefoucauld, de Retz, Saint-Simon et Massillon.

Comme je l'ai déjà constaté ailleurs, en parlant de Staaf ${ }^{24}$, son principe de sélection d'auteurs semble être celui de l'exhaustivité. Il donne donc des échantillons d'un grand nombre d'auteurs et pour ne pas sombrer dans un nombre de pages excessif, ces échantillons ont forcément des dimensions plus modestes que ceux de Dubb. Les prosateurs et les poètes de quelque envergure occupent par conséquent seulement environ 150 des 250 pages consacrées à l'époque classique. Le gagnant dans cette course de popularité posthume est Racine, avec des fragments d'Andromaque, d'Iphigénie, de Phèdre, d'Esther et surtout d'Athalie, de la poésie religieuse, mais aussi de la prose: ses éloges de Corneille et de Louis XIV et des lettres à son fils; le tout compris dans l'espace de moins de vingt pages. Après lui, il y a Fénelon, toujours, mais Télémaque est cette fois mis de côté, au bénéfice de L'Existence de Dieu, des Dialogues des morts, même des fables et des lettres. Molière est le troisième, avec la prose et la poésie: petits fragments de L'Avare, du Bourgeois Gentilhomme, du Malade imaginaire, des Fâcheux, du Misanthrope, de Tartuffe et des Femmes savantes. Puis vient Boileau, toujours de tout petits fragments, des Embarras de Paris, des Epîtres, de L'Art Poétique et du Lutrin. La Fontaine est représenté par 12 fables, Bossuet, aussi fragmenté, par ses Oraisons funèbres et ses Sermons, finalement La Bruyère par 15 Caractères. D'autres prédicateurs que Bossuet ont trouvé leur place, comme Massillon et Bourdaloue, et on n'a pas oublié Madame de Sévigné, ni Madame de La Fayette, le cardinal de Retz, Saint-Simon et La Rochefoucauld. Les contes de Perrault auraient pu faire contrepoids aux prédicateurs, mais il ne figure que comme auteur d'un poème, Portrait de l'amitié. Sont-ils trop enfantins ou sont-ils trop difficiles à fragmenter?

17 Pour cette période aussi, de 1840 à 1870 , je ne peux comparer que ce qu'on a étudié dans les écoles de la capitale. Parmi ces trois grands propagateurs, que je viens de décrire, il n'y a qu'un seul cité dans les rapports, et ceci dans une seule école. Il s'agit de Staaf, qui, contre toute attente, est utilisé comme livre de débutants dans l'école expérimentale de l'État ${ }^{25}$. Mais, comme toujours, il n'est pas absolument sûr que l'époque ait été le sujet d'une étude. On parle bien du premier tome, ce qui est rare, mais ce n'est que la première moitié de ce tome qui traite l'époque classique; le deuxième se consacre au $18^{\mathrm{e}}$ siècle. L'absence de Caravello dans les rapports est explicable par le fait qu'il est imprimé à Goteborg, la deuxième ville de Suède, située sur la côte ouest. Il est vrai, cependant, que cet ouvrage n'a pas pu jouer un rôle considérable, puisqu'il n'y a jamais eu d'édition ultérieure. Dubb, imprimé à Lund, en Scanie, a dû régner dans le sud de la Suède, car, un peu plus tard, quand on a la possibilité de se faire une image de la situation dans toute la Suède (malheureusement seulement pour les écoles de garçons), il tient une des places en tête, surtout grâce aux écoles des régions méridionales. A Stockholm, dans les écoles publiques, on s'en tient à Staaf, mais sans doute en étudiant des textes plus modernes. On continue aussi à utiliser les anthologies d'Elfving, de Paban (Beautés...) et de Gruner-Wildermuth ${ }^{26}$, ce dernier contenant une partie minime d'auteurs classiques, et une anthologie imprimée en Suisse, la Chrestomathie française, par A. Vinet ${ }^{27}$. Celle-ci contient un certain nombre de textes venant de l'époque qui nous intéresse, surtout des oraisons (Bossuet, Massillon et Pellisson) et des scènes d'Athalie de Racine. Une année, une seule (1856), on annonce avoir travaillé Iphigénie dans le lycée de Stockholm, à côté des Beautés de Paban. Les écoles privées de la ville, se servent aussi des Beautés, mais l'une d'elles dit 
avoir fait travailler Molière (Tartuffe et L'Avare) ${ }^{28}$. La seule école de filles à donner des indications, l'école de Wallin, semble avoir laissé de côté l'étude des classiques et se concentre sur le $19^{\mathrm{e}}$ siècle $^{29}$. A en juger par ces résultats, l'étude des auteurs classiques décline pendant cette deuxième période, par rapport à la période précédente.

L'édition de la troisième période, 1870-1890, présente une autre image :

Publication d'un ouvrage plus ou moins complet :

\begin{tabular}{|c|c|c|}
\hline $\begin{array}{l}\text { Avant l'époque } \\
\text { classique }\end{array}$ & - & \\
\hline $\begin{array}{l}\text { L'époque } \\
\text { classique }\end{array}$ & 2 éditions & Molière, L'Avare, Fénelon, Telemaque \\
\hline $18^{\mathrm{e}}$ siècle & $\begin{array}{l}2 \\
\text { éditions. }\end{array}$ & Florian, Guillaume Tell; Voltaire, Histoire de Charles XII; \\
\hline $19^{\mathrm{e}}$ siècle & $\begin{array}{l}13 \\
\text { éditions. }\end{array}$ & $\begin{array}{l}\text { Guizot, Rydberg }{ }^{30} \text {, Banville, Scribe, Girardin, Daudet, Souvestre, } \\
\text { Mérimée, Mignet. }\end{array}$ \\
\hline Total: & $\begin{array}{l}17 \\
\text { éditions }\end{array}$ & \\
\hline \multicolumn{3}{|c|}{ Anthologies et livres de lecture: } \\
\hline & $\begin{array}{l}25 \\
\text { éditions }\end{array}$ & $\begin{array}{l}10 \text { éditions contiennent des textes de l'époque classique, dont } 5 \\
\text { dans des proportions surpassant } 1 / 10 \text { du volume. }\end{array}$ \\
\hline
\end{tabular}

Si la période précédente, dans les débuts tâtonnants d'une instruction nationale, favorise le livre de lecture ou l'anthologie (quatre fois plus de ce genre de publications que d'éditions d'ouvrages), les proportions commencent, dans la période actuelle des mises en cause des expériences, à redevenir à peu près égales. Pour les éditions $d^{\prime} œ u v r e s ~ d u ~ 17^{e}$ siècle, Molière et Fénélon tiennent bon. L'une des anthologies est la $3^{\mathrm{e}}$ édition de Dubb, où Molière occupe une partie importante; et une autre, Klint (1877) commence par Le Bourgeois Gentilhomme. On dirait qu'à la longue, Molière a gagné la partie.

21 Mais dans les trois autres, avec un pourcentage satisfaisant de textes provenant de l'époque classique, un auteur jusqu'ici inconnu dans ce contexte prend le dessus: Perrault. Il s'agit du premier tome, pour débutants, de Bergman ${ }^{31}$ (1887) et des deux éditions de Schrevelius ${ }^{32}(1888,1889)$, aussi pour débutants. Quelle est l'explication de ce phénomène? Qui ose couper aussi radicalement avec la tradition? La réponse est assez simple: après le grand débat des années 1880 , sur les méthodes d'apprentissage des langues vivantes, où onprogresse vers les méthodes plus naturelles et plus «directes» et où l'on condamne les exercices de phrases isolées et la langue sans contexte, un proviseur d'une école mixte (Bergman) et une enseignante d'un lycée de filles (Schrevelius) trouvent tous les deux que les contes, avec un contenu simple et intéressant pour les enfants et la jeunesse, pourraient constituer un texte convenable. On dirait qu'il y a eu concertation, car les contes ne sont pas les mêmes: Les Fées, La Belle au bois dormant, Cendrillon pour Bergman; et Le petit Chaperon rouge, Le Chat 
botté, Le Petit Poucet pour Schrevelius. Même dans l'édition, on peut donc constater une influence de la présence grandissante des filles dans le corps des élèves du pays; il est remarquable, il me semble, que malgré les discussions générales, les auteurs de manuels tournés vers l'école publique des garçons n'aient pas osé agir en conséquence quant aux idées nouvelles: donner aux élèves des textes enfantins, peut-être «trop» simples. Pour l'éducation des filles, l'idée d'utiliser, pour les apprenants de langues, des textes destinés à un âge beaucoup plus tendre que l'âge des élèves en question, date au moins du milieu de $18^{\mathrm{e}}$ siècle où Madame Leprince de Beaumont déclare dans la préface de son Magazin des enfans;

Le dégoût de la plupart des enfans pour la lecture vient de la nature des livres qu'on leur met entre les mains; ils ne les comprennent pas, et delà naît inévitablement l'ennui. [...] Une fille de quinze ans qui commence à apprendre le français a besoin d'un style aussi simple qu'une autre de cinq ans qui lit dans sa langue maternelle. (Leprince de Beaumont 1820: 2-3)

Voilà pour l'édition. Quelle a été la situation de la lecture réelle, dans les écoles de filles et les écoles de garçons? Pour les écoles de filles, je ne dispose, comme auparavant, que de la documentation de celles de Stockholm, sauf pour la dernière année; pour les écoles de garçons, en revanche, il existe des listes nationales des manuels utilisés dans toutes les matières, l'une pour l'année 1876/77, l'autre pour 1889/9033. Parmi les écoles de filles ou les écoles mixtes de Stockholm, on étudiait, dans les années 1870, Athalie de Racine (4 écoles), les fables de La Fontaine (2 écoles), Esther de Racine (une école) et, chose curieuse, Télémaque de Fénelon dans une école, donc avant l'édition scolaire tardive de cet ouvrage, en $1882^{34}$. A l'échelle nationale, pour les garçons, on s'est servi, au cours de la même décennie, tout particulièrement de l'anthologie parmi les plus «classiques», Dubb (10 écoles), à l'époque arrivé à sa $3^{e}$ édition. Six écoles ont travaillé la troisième partie de la Nouvelle bibliothèque française à l'usage de la jeunesse (1861) de L.E. Paban, qui contient un texte de Rodolphe Tôpffer. mais aussi L'Avare et le second acte du Bourgeois Gentilhomme de Molière. Son Malade imaginaire ${ }^{35}$ a été étudié dans deux écoles, ce qui est aussi le cas pour Athalie de Racine, qui finalement n'a donc pas été considérée comme une pièce pour filles seulement. Pour comparer, on se sert d'une anthologie sans auteurs classiques (Berndston, Variétés littéraires, 1851-1884) dans 14 écoles, de l'Histoire de Charles XII de Voltaire dans huit écoles, de textes de Souvestre dans six écoles et de Scribe dans trois, sans compter les autres. Et les lecteurs de Dubb ont bien pu se pencher sur les textes du $18^{\mathrm{e}}$ siècle et pas beaucoup sur ceux $\mathrm{du} 17^{\mathrm{e}}$. Le résultat me paraît assez maigre. Il est encore plus maigre une quinzaine d'années plus tard, 1889/90. Dubb n'est cité que pour une seule école, L'Avare figure dans deux, mais Perrault, par contre, a été accepté même dans les écoles de garçons, puisque huit écoles annoncent cet ouvrage, qui contient aussi, ne l'oublions pas, des fables de La Fontaine. Les écoles de filles reculent aussi. Sauf quelque pièce de Molière dans Tune, des fables de La Fontaine dans l'autre, et ce Télémaque obstiné, elles se sont tournées, à l'échelle nationale, selon l'historienne des écoles de filles du $18^{\mathrm{e}}$ siècle en Suède. Gunhild Kyle, presque exclusivement vers la littérature du $19^{\mathrm{s}}$ siècle $^{36}$.

Regardons finalement la dernière période, 1890-1900:

\section{Publication d'un ouvrage plus ou moins complet :}




\begin{tabular}{|c|c|c|}
\hline $\begin{array}{l}\text { Avant } \\
\text { l'époque } \\
\text { classique }\end{array}$ & - & \\
\hline $\begin{array}{l}\text { L'époque } \\
\text { classique }\end{array}$ & - & \\
\hline $18^{\mathrm{e}}$ siècle & - . & \\
\hline $19^{\mathrm{e}}$ siècle & $\begin{array}{l}31 \\
\text { éditions. }\end{array}$ & $\begin{array}{l}\text { Verne, Vacquerie, Coppée, Erckmann-Chatrian, Daudet, Berthet, Blondel } \\
\text { Halévy, Normand, Conti, Labiche, Theuriet, Richebourg, Champo] } \\
\text { Girardin; Mmes de Bawr, Foa, de Pressensé, Colomb, de Witt; Souvestre, } \\
\text { Porchat, Musset, Claretie, de Maupassant, Colomb, Legouvé, Mérimée } \\
\text { Ohnet, Sardou, Duruy, Malot, Augier/Sandeau, Carnoy, Hait, Feuillet, } \\
\text { Laboulaye. }\end{array}$ \\
\hline Total: & $\begin{array}{l}31 \\
\text { éditions }\end{array}$ & \\
\hline \multicolumn{3}{|c|}{ Anthologies et livres de lecture: } \\
\hline & $\begin{array}{l}13 \\
\text { éditions }\end{array}$ & $\begin{array}{l}\text { Aucune édition ne contient de textes de l'époque classique, dans des } \\
\text { proportions surpassant } 1 / 10 \mathrm{du} \text { volume. Deux seulement présentent } 5 \\
\text { pages de Fénelon (PA Norstedt \& Soners skolbibliotek, 3e partie, } 1891 \text {, } \\
\text { 1898).. }\end{array}$ \\
\hline
\end{tabular}

On dirait, en voyant ce tableau, qu'au cours de la dernière décennie du siècle passé, il y a exactement cent ans, l'étude des auteurs classiques était définitivement terminée dans les écoles suédoises, du moins pour le siècle en question. En regardant une troisième liste nationale sur les manuels utilisés dans les écoles de garçon ${ }^{37}$, on dirait la même chose. Une seule école se sert de Bergman (contes de Perrault), pour le reste, c'est le nouvel ordre qui règne, celui du mouvement de la Réforme, qui préconise des textes faciles, avec la langue contemporaine, de préférence autour des choses pratiques de tous les jours. Mais si l'on regarde sur l'échelle locale de Stockholm, l'image change un peu. Il est vrai que les écoles de garçons, qu'elles soient publiques, expérimentales, privées ou mixtes, ont complètement abandonné l'époque classique. La dernière année scolaire du siècle, cependant, trois des quatre écoles de filles les plus importantes de la capitale arborent toujours le drapeau du classicisme. Tout d'abord dans les écoles bien établies, fondées dans les années 1830 et 1840: dans celle de Wallin, on travaille L'Avare et Iphigénie ${ }^{38}$, dans celle d'Âhlin, Le Bourgeois Gentilhomme ${ }^{39}$. Mais ce qui est le plus surprenant, c'est que dans une école nouvelle, créée à la naissance du mouvement de la Réforme, par une femme qui non seulement se ralliait à ce mouvement, mais était la fondatrice et la rédactrice de la revue Verdandi, revue qui servait de porte-parole du mouvement et de centre d'information des aclivités de la société inspirée par Viëlor, Quousque tandem, dans cette école, les jeunes filles ont lu L'avare, Les précieuses ridicules et Iphigénie ${ }^{40}$.

Comme toujours, les conclusions sont difficiles à tirer. Les filles, ou leurs éducatrices, sont, nous l'avons vu, les instigatrices d'un ordre nouveau, où l'on recommande la méthode directe et la lecture de textes faciles, contemporains et intéressants pour les 
jeunes, et en même temps les conservatrices d'une époque classique en voie de disparition. Comment expliquer ce phénomène? Une faible indication pourrait être que les écoles de filles, selon Kyle, avaient une tendance plus marquée à lire des ouvrages entiers, pas des anthologies, qu'elles montraient aussi un plus grand esprit d'indépendance que les écoles de garçons ${ }^{41}$. D'après mes propres expériences, il semble que les filles lisaient également en moyenne plus de texte, que les garçons. Mais ce n'est pas toute l'explication, et pour la trouver, il faudrait des recherches plus poussées. Toujours est-il que le résultat des recherches que je viens de communiquer montrent, s'il fallait le prouver encore une fois, que l'histoire de l'éducation des filles et celle de l'éducation des garçons ne se ressemblent pas, même dans le domaine apparemment neutre de la lecture des auteurs de l'époque classique.

\section{NOTES}

1. Je signale que je prends «l'époque classique» dans le sens strict du terme, c'est-à-dire le siècle de Louis XIV. Ceci implique que je ne traiterai ni Corneille, ni Voltaire.

2. Pour l'édition des manuels de français en Suède du début (1626) jusqu'en 1905, voir Hammar, E. (1980): L'enseignement du français en Suède jusqu'en 1807. Méthodes et manuels, Stockholm, Akademilitteratur, 167-188; et Hammar, E. (1985): Manuels de français publiés à l'usage des Suédois de 1808 à 1905, Acta Bibliothecae Regiae Stockholmiensis XLIV, Stockholm, Norstedts. Par la suite, les calculs sur l'édition du $19^{\mathrm{e}}$ siècle seront faits sur la base de ce dernier livre, où l'on trouvera également les détails quant aux ouvrages concernés.

3. La suivante publication scolaire suivante de Télémaque en Suède pendant le siècle n'est que de 1882.

4. Voir Hammar, E. (1995): «De Télémaque à la Réforme. L'institutionnalisation du français dans le gymnasium de Stockholm 1824-1905», Documents pour l'histoire du français langue étrangère ou seconde, n. ${ }^{\circ} 15,1995$, pp. 9-10.

5. Je parle d'éditions plutôt que d'ouvrages, pour donner une idée de la diffusion, d'ailleurs difficile à établir.

6. Les auteurs sont cités, comme par la suite, dans l'ordre chronologique de leur publication. Notons que certains ouvrages publiés en français sont des traductions.

7. Puisqu'il m'est impossible de savoir, dans la plupart des cas, quelles pages d'une anthologie ou d'un livre de lecture ont été utilisées dans les écoles, il me semble plus prudent de ne traiter que les livres présentant les auteurs classiques dans des proportions suffisamment importantes.

8. Gedike, F: Doct. Fr. Gedikes fransyska läsebok for nybegynnare.

9. Moberg, P. \& Wallman, C. G.: Recueil de morceaux intéressants.

10. Svedelius, P. G.: Fransysk och swensk läsebok.

11. Paban, L. E.: Beautés de la littérature française. Pour le contenu et l'organisation de cette anthologie, qui a l'ambition de suivre «le Cours de littérature comparée, adopté par l'Université Royale de France», voir Hammar, E. (1995): «Du texte édifiant à la littérature. Le choix de lecture française jusqu'en 1860 en Suède», Regards sur l'histoire de l'enseignement des langues étrangères (éd. H. Christ/G. Hassler),Tiibingen, Gunter Narr, pp. 110-117. 
12. Les renseignements sur le lycée et les écoles publiques de Stockholm ont été trouvés, pour la plupart, dans les «listes de cours» et des «livres de leçons» inédits, aux Archives de la ville de Stockholm, cotes A I b et C VIII b.

13. Hall, B. R. (1935): Manliga privätlaroverk, Ärsbócker i svensk undervisnings historia 44, Lund, pp. 103-110.

14. Ericsson, H. (1885): Hillska skolan a Barnängen 1830-1846, Stockholm, pp. 102-109.

15. (1875): Redogörelse, Stockholm, Bibliothéque Royale, non catalogué.

16. (1888): Undersökning af Sveriges högre flickskolor, Stockholm, p. 328.

17. Elfwing, J. A.: Fransk läsebok för skolor och gymnasier [...] Sednare afdelningen: Ouvrages dramatiques.

18. Paban, L. E.: Nouvelle bibliothèque française [...] Troisième livraison,

19. Pauli, C. G.: Fransk läsebok med kommentarier. Sednare häftet

20. Caravello, H. C. T.: Edda française des $17^{e}$ et $18^{e}$ siècles.

21. Dubb, P. J. C: Handbok i franska spräket och litteraturen.

22. Staaf, F. N.: Urval ur franska litteraturen [...j Förra delen. (Intill Franska revolutionen.)

23. Pour une présentation de cette ambition, voir Hammar, 1995, ouvr. cité

24. Voir Hammar, 1995.

25. (1866): Nya Elementarskolan i Stockholm. Ärsberättelse fàr läsedret 1865-1866.Stockholm, Bibl.Royale, non catalogué

26. Gruner, F. S. \& J.D. Wildermuth, J. D. $(1857,1858)$ : Fransk chrestomathie. Stockholm, Ed. F. L. Samuelsson.

27. Vinet, A. (1844): Littérature de l'adolescence. Choix de morceaux pris dans les différentes branches de l'art d'écrire et tirés des meilleurs écrivains français, 4 ééd., Bale, Neukirch.

28. (1857): Lärokurser for Privata Nya Elementarskolan à Norr, i Stockholm, Stockholm, Bibl. Royale, non catalogué.

29. (1867): Wallinska skolans lärokurser. Stockholm. Bibl. Royale, non catalogué.

30. Il s'agit d'un conte d'un auteur suédois, Rydberg, V. (1876): La Veille de Noël du petit Vigg, Stockholm, qui a été traduit comme exercice de français dans une école de Stockholm et puis imprimé.

31. Bergman, C. G.: Berättelser affranska författare.

32. Schrevelius, A.: Fransk läsebok for nybegynnare. Le texte de Perrault dans cet ouvrage est modernisé

33. (1881): Förtekning pâ de böcker, som varit begagnade vid undervisningen [...] under läseäret 1876-77 [...] upprättad af D:r Bernhard Lunestedt, Stockholm, et (1891): Förteckning ofver de arbeten, som varit begagnade för undervisningen [...] under läseäret 1889-1890 [...]. Utarbetad af G. Eneström, Stockholm.

34. Les renseignements ont été trouvés, pour les écoles de filles, dans (1879): Lärokurser, läro- och läseböcker vid Högre Elementarläroverket for flickor iStockholm, Stockholm; (1873): Undervisningsplan och lärokurser vid Institut de langues modernes (pour des jeunes filles) i Stockholm, Stockholm; (1877): Tills vidare gällande lärokurser vid Statens normalskola för flickor, Stockholm, tous à la Bibl. Royale, non catalogués; pour l'école mixte, Beskowska skolan aux Archives de la ville de Stockholm, «Kurrser genomgängna 1867-1875».

35. I1 s'agit d'une édition étrangère, puisque cet ouvrage n'a pas été publié en Suède.

36. Voir Kyle, G. (1972): Svensk flickskola under 1800-talet, Ârsböcker i svensk undervisningshistoria 128, Stockholm, pp. 247-252.

37. (1902): Redogörelse for de arbeten, som varit begagnade för undervisningen [...] under läsearet 1899-1900. [...] Utarbetad of K.H. Karlsson, Stockholm.

38. (1900): Redogörelse för Wallinska skolan [...\} 1899-1900. Stockholm, Bibl. Royale, non catalogué.

39. (1900): Ârsredogörelse för arbetet inom Âhlinska skolan i Stockholm /.../ 1899-1900, Stockholm, Bibl. Royale, non catalogué. 
40. (1900): Redogörelse för Anna Sandstroms skolan läs-âret 1899-1900, Stockholm, Bibl. Royale, non catalogué.

41. Kyle, (1972): ouvr. cité, p, 171

\section{AUTEUR}

\section{ELISABET HAMMAR}

Université de Linkôping 